\title{
¿Previene la actividad física y mental el deterioro cognitivo? Evidencia de la investigación animal
}

\section{Does physical and mental activity prevent cognitive impairment? Evidence from animal research}

\author{
Patricia Sampedro-Piquero y Azucena Begega \\ Laboratorio de Psicobiología, Facultad de Psicología, Universidad de Oviedo, España \\ Disponible online 31 de diciembre de 2013
}

\begin{abstract}
En las últimas décadas se han desarrollado varias estrategias farmacológicas para prevenir el declive cognitivo en el envejecimiento, sin embargo la inefectividad de la mayoría de ellas ha hecho que las intervenciones conductuales estén recibiendo cada vez más atención. La estimulación cognitiva y la actividad física han mostrado importantes beneficios al reducir las alteraciones cognitivas relacionadas con la edad y la demencia. Gracias a los modelos animales cada vez se sabe más acerca de los mecanismos neurobiológicos que subyacen a la reserva cognitiva y cerebral que promueven estas intervenciones. En esta revisión presentaremos varias investigaciones en roedores viejos en las que se muestran los efectos positivos del enriquecimiento ambiental y ejercicio aeróbico sobre la función cerebral y cognitiva de estos animales. La implicación de estas investigaciones para el envejecimiento humano será también discutida.

Palabras clave: Enriquecimiento Ambiental; Ejercicio Aeróbico; Envejecimiento; Enfermedades Neurodegenerativas; Modelo Animal.

In recent decades, several pharmacological strategies have been developed to prevent age-related cognitive impairment. However, the ineffectiveness of the majority of these strategies has led to growing interest in behavioural intervention. Cognitive stimulation and physical activity have been shown to provide significant benefits by counteracting cognitive impairment and dementia. Animal models have provided information on the neurobiological mechanisms that mediate the formation of cognitive and brain reserve. This paper reviews several studies on aged rodents showing the positive effects of environmental enrichment and aerobic exercise on brain and cognitive function of these animals. The implications of this research for human aging is discussed.
\end{abstract}

Keywords: Environmental Enrichment; Aerobic Exercise; Aging; Neurodegenerative Diseases; Animal Model.

Correspondencia: Patricia Sampedro-Piquero. Laboratorio de Psicobiología, Facultad de Psicología (Universidad de Oviedo). Plaza Feijoo s/n 33003 , Oviedo, Asturias, España. Fax: (+34) 985104144. Email: uo179677@uniovi.es. E-mail de la co-autora: begega@uniovi.es 
En los últimos años ha crecido el interés sobre cómo el ambiente que nos rodea y nuestras experiencias pasadas pueden modular nuestro cerebro. Este interés se ha dado sobre todo en el campo del envejecimiento y las enfermedades neurodegenerativas debido al hallazgo de que una vida mentalmente activa puede prevenir el deterioro cognitivo. Variables como los años de educación, la actividad profesional y la frecuencia de actividades mentales, físicas, sociales y recreativas pueden predecir la probabilidad de una futura demencia. Este fenómeno es referido como "reserva cognitiva y cerebral" y surge sobre todo a partir de estudios epidemiológicos en los que se encuentra que una actividad mental, frecuente y compleja, parece protegernos de la manifestación clínica del daño cerebral (Stern, 2009). Los mecanismos cerebrales que subyacen a esta reserva se han ido conociendo gracias a estudios con modelos animales y el empleo del Enriquecimiento ambiental (EA). El EA es un paradigma experimental que consiste en modificar la condición de vivienda de los roedores incluyendo en su modo de vida factores como la exposición a novedad y la estimulación tanto física, social, sensorial, como cognitiva. De este modo, los animales conviven en grupo en grandes jaulas, con diferentes objetos como plataformas, ruedas, cuerdas, materiales de madera o pelotas que son cambiados frecuentemente. Aunque variables como el nivel educativo y las actividades profesionales y de ocio son imposibles de extrapolar de humanos a animales, la exposición a EA ha mostrado mejorar las funciones cognitiva, social y física, como ocurre en el caso de un estilo de vida social e intelectualmente activo en humanos. En esta revisión exploraremos la repercusión que el EA tiene sobre el cerebro y cognición, tanto durante el envejecimiento sano como en modelos de enfermedades neurodegenerativas. También revisaremos el beneficio del ejercicio aeróbico como uno de los componentes clave del EA.

\section{Actividad mental y riesgo de demencia}

El proceso de envejecimiento provoca un declive de diferentes funciones cognitivas tanto en humanos como animales. Sin embargo, la participación en un estilo de vida activo, a través de actividades que promuevan la estimulación mental, puede ayudar a reducir el riesgo de demencia y constituir una medida prometedora de salud pública (Navarro-González, Calero-García, Pérez-Díaz, y Gómez-Ceballos, 2008; Valenzuela y Sachdev, 2006, 2009). El nivel educativo es uno de los factores más estudiados debido a su relación con el declive cognitivo que ocurre en el envejecimiento (Katzman, 1993). Se ha demostrado que aunque la educación no previene el inicio de Alzheimer, quizás proporciona protección contra la manifestación clínica de sus síntomas (Snowdon et al., 1996). En sujetos con alto nivel educativo el grado de deterioro cerebral que acompaña a una demencia como el Alzheimer es mucho más severo que en casos de bajo nivel educativo, pero curiosamente las alteraciones cognitivas que presentan son mucho más leves. Por ejemplo, enfermos de Alzheimer con alto nivel educativo tuvieron un déficit de perfusión parietotemporal más severo, indicando que la enfermedad a nivel cerebral estuvo más avanzada, a pesar de que sus síntomas clínicos pareciesen indicar lo contrario (Stern, Alexander, Prohovnik y Mayeux, 1992). De este modo, y de acuerdo a esta evidencia, dos sujetos con un similar deterioro a nivel cerebral pueden presentar un rendimiento cognitivo totalmente diferente (Bennet et al., 2003; Kemppainen et al., 2008). Los estudios post-mortem también confirman la hipótesis de la reserva al encontrar signos neuropatológicos propios de la demencia de Alzheimer, como ovillos neurofibrilares o placas seniles, en cerebros de personas mayores que nunca tuvieron deterioro cognitivo (Katzman et al., 1988; Neuropathology Group, 2001). Sin embargo, en el momento en el que los síntomas clínicos aparecen, los sujetos con alto nivel educativo muestran un declive mucho más rápido, quizás debido a que el grado de patología cerebral ya se encuentra muy avanzada (Fig.1). Todos estos datos proporcionan la base de la hipótesis conocida como reserva cognitiva y cerebral que explicaremos a continuación (Stern, 2002, 2009).

\section{Figura 1}

Ilustración de cómo la reserva cognitiva media entre la patología de la enfermedad de Alzheimer y su expresión clínica. En casos de alta reserva cognitiva, el diagnóstico clínico de demencia aparece más tarde cuando ya la patología cerebral es muy severa. En el momento en que en personas con alta reserva cognitiva aparecen los síntomas clínicos, la progresión de la enfermedad será mucho más rápida.

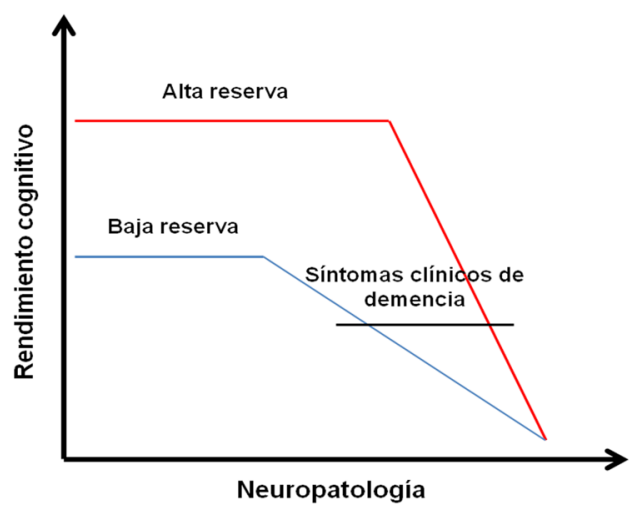

\section{El constructo de reserva}

El constructo de reserva ha sido propuesto para dar cuenta de la falta de relación entre el grado de daño cerebral y la manifestación clínica del paciente. Dentro del concepto de reserva podemos distinguir entre reserva cognitiva y reserva cerebral, siendo ambas no excluyentes y participando por igual en proporcionarnos protección contra enfermedades neurodegenerativas. La principal diferencia entre ambas es el carácter activo o pasivo que las constituye. La reserva cerebral es un ejemplo de reserva pasiva en donde diferencias individuales en el tamaño cerebral, número de neuronas, sinapsis, o de ramificaciones 
dendríticas permite al sujeto enfrentarse mejor a la patología cerebral. Varios estudios encontraron que la prevalencia de demencia fue menor en sujetos con cerebros mayores que en aquellos con cerebros más pequeños, quizás porque los primeros son capaces de soportar durante más tiempo la patología antes de que los síntomas aparezcan (Schofield, Logroscino, Andrews, Albert y Stern, 1997). Por el contrario, la reserva cognitiva es una forma activa de reserva en la que es mucho más importante el funcionamiento cerebral que su estructura. De este modo, nuestro cerebro intentaría enfrentarse contra la patología cerebral mediante la puesta de marcha de mecanismos compensatorios o formas de procesamiento cognitivo más eficientes (Stern, 2002). El desarrollo de estas reservas va a depender de nuestra experiencia a lo largo de la vida, incluyendo como vimos nuestro nivel educativo, pero también, el apoyo emocional, nuestra ocupación, actividades de ocio, o la realización frecuente de ejercicio moderado (Richards y Deary, 2005; Scarmeas y Stern, 2003).

Una de las principales limitaciones de la teoría de la reserva cognitiva es la laguna que existe entre el concepto y su sustrato neurobiológico (Richards y Deary, 2005). La investigación en modelos animales resulta útil para comprender la influencia de una vida activa sobre el deterioro cognitivo relacionado con el envejecimiento, a la vez que pueden aportar información acerca de los mecanismos cerebrales implicados en el efecto beneficioso de vivir en un ambiente estimulante (Petrosini et al., 2009). Estudios preclínicos acerca de cómo el cerebro responde a experiencias estimulantes han empleado con frecuencia el paradigma experimental del EA, e investigadores como Daffner (2010), han enfatizado la importancia de la investigación básica en animales para identificar qué factores promueven un envejecimiento saludable. En general, los animales mantenidos en ambientes enriquecidos muestran una menor velocidad de progresión de alteraciones cognitivas, así como cambios neurobiológicos que indican la presencia de mayor plasticidad cerebral (Nithianantharajah y Hannan, 2006).

\section{Enriquecimiento ambiental}

El primero en darse cuenta de los beneficios del EA fue Donald Hebb (1947) el cual observó que aquellas ratas que él tenía como mascota y que permitía que explorasen libremente su casa rendían mejor en pruebas de memoria en comparación a las que permanecían en condiciones estándar de laboratorio. Posteriormente, en la década de los 60, Rosenzweig et al. (1962) introdujeron el EA como un paradigma científico que mostraba efectos positivos sobre la capacidad de aprendizaje y memoria de los animales. En este ambiente, diferentes objetos como túneles, juguetes, plataformas o cuerdas son introducidos con el fin de estimular la conducta exploratoria de los animales (Fig.2). El EA ofrece la posibilidad de aumentar la interacción social, la estimulación cognitiva y sensorial, así como la capacidad de resolución de problemas. En respuesta a esta estimulación el cerebro experimenta gran cantidad de cambios, tanto en su estructura como funcionamiento, con el objetivo de enfrentarse mejor a las demandas ambientales (Leggio et al., 2005; Mandolesi et al., 2008; Sale, Berardi y Maffei, 2009).

\section{Figura 2}

Típico ambiente enriquecido en el que se ofrece estimulación motora, sensorial, cognitiva y física a los roedores. Los objetos son frecuentemente cambiados para motivar las conductas exploratorias en los animales (Sampedro-Piquero, Zancada-Menéndez, C., Begega A., Rubio, S. y Arias, J.L., 2013).

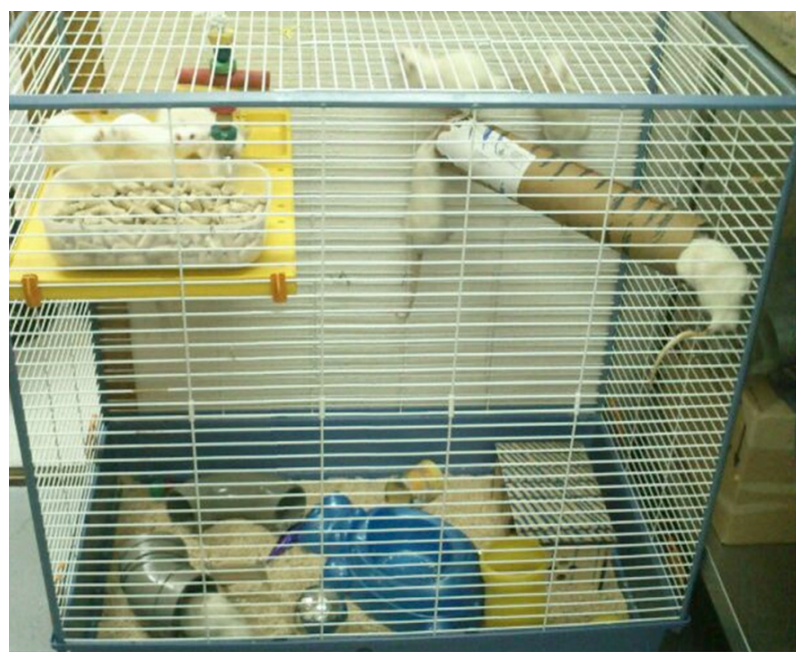

Muchos trabajos en roedores han sido publicados acerca de la influencia del EA sobre los cambios que ocurren con la edad tanto a nivel cognitivo como fisiológico. A continuación resumiremos los resultados más interesantes acerca del potencial terapéutico del EA, tanto durante el proceso de envejecimiento normal como en modelos animales de enfermedades neurodegenerativas.

\section{Beneficios del EA en roedores viejos}

Aunque un breve período de exposición a EA (unas pocas semanas) es capaz de reducir los déficits en aprendizaje y memoria en roedores viejos (Bennet, McRae, Levy y Frick, 2006; Harburger, Lambert y Frick, 2007), otros han encontrado que una exposición durante toda la vida puede tener un efecto más potente (Kobayashi, Ohashi y Ando, 2002). A nivel conductual, los roedores que viven en condiciones de EA presentan un mejor rendimiento en pruebas de memoria, tanto espacial como no espacial, en comparación a las que viven en condiciones estándar de laboratorio (Bennet et al., 2006; Diniz et al., 2010; Kobayashi et al., 2002). Igualmente, este beneficio no solo ocurre sobre la memoria espacial, sino también sobre la precisión de respuesta en una prueba de atención sostenida (Harati et al., 2011) o en funciones ejecutivas como la capacidad de flexibilidad y alternancia (De Bartolo et al., 2008). Los roedores que reciben EA, tanto jóvenes como viejos, tam- 
bién presentan una adaptación mucho más rápida a ambientes nuevos, lo cual podría estar reflejando un procesamiento más eficiente del contexto que les rodea permitiéndoles habituarse antes a él (Zimmermann, Stauffacher, Langhans y Wurbel, 2001). Por último, también se ha visto que en pruebas clásicas de medida de ansiedad no condicionada, como el laberinto elevado en cero, o el laberinto elevado en cruz, estos animales son menos ansiosos permaneciendo más tiempo y explorando más las áreas abiertas de estos laberintos (Chorpita y Barlow, 1998).

Todos estos beneficios conductuales se encuentran relacionados con cambios en las respuestas fisiológicas y neurobiológicas de estos animales. A nivel estructural, se ha encontrado aumento de peso cerebral, de ramificaciones dendríticas o del número de sinapsis en varias regiones cerebrales, principalmente en el hipocampo y corteza cerebral, que son de las más vulnerables con la edad (Nithianantharajah y Hannan, 2006). El EA estimula la neurogénesis a la vez que promueve un aumento de plasticidad neuronal en el hipocampo (Castilla-Ortega, Pedraza, Estivill-Torrús y Santín, 2011; Kempermann, Gast y Gage, 2002), lo cual incrementaría el número de receptores para diferentes neurotransmisores y elevaría la presencia de factores neurotróficos como BDNF, NGF y GDNF entre otros (Mohammed et al., 2002; Mora, Segovia y del Arco, 2007; Li, Niu, Jiang y Hu, 2007). En la actualidad, hay numerosos estudios que sugieren que la neurogénesis hipocampal adulta está envuelta en funciones cognitivas y emocionales dependientes del hipocampo (Kempermann, 2008). Así, aquellos roedores que muestran una mayor capacidad neurogénica presentan también un mejor rendimiento en pruebas de memoria espacial, llegando a encontrarse una relación entre el nivel de aprendizaje alcanzado y el número de nuevas neuronas (Sisti, Glass y Shors, 2007). En cuanto a la relación con procesos emocionales, las hormonas relacionadas con respuestas de ansiedad disminuyen la neurogénesis y además esta es necesaria para el efecto positivo de los antidepresivos sobre el estado anímico (Petrik, Lagace y Eisch, 2012).

Otros cambios descritos en el cerebro de ratas enriquecidas es el aumento de gliogénesis (Williamson, Chao y Bilbo, 2012), cambios en los potenciales sinápticos excitatorios (Mohammed et al., 2002) y también en la potenciación a largo plazo, conocido como el mecanismo fisiológico que subyace a ciertos procesos de aprendizaje y memoria (Van Praag, Kempermann y Gage, 2000).

Todos estos cambios podrían estar relacionados con la formación de la reserva cognitiva (Fig.3). Además, permitirían el establecimiento de nuevas redes cerebrales que participarían en un procesamiento más eficiente y en una mayor capacidad de resolución de problemas, además de compensar el funcionamiento de redes neuronales alteradas por el envejecimiento.
Figura 3

Esquema que muestra la relación el efecto del EA y el proceso de envejecimiento sobre la plasticidad cerebral. La interacción, entre los cambios positivos inducidos por el EA y los negativos del envejecimiento, se manifestaría en el funcionamiento cognitivo y conductual a causa de los cambios que se producen en las redes neuronales.

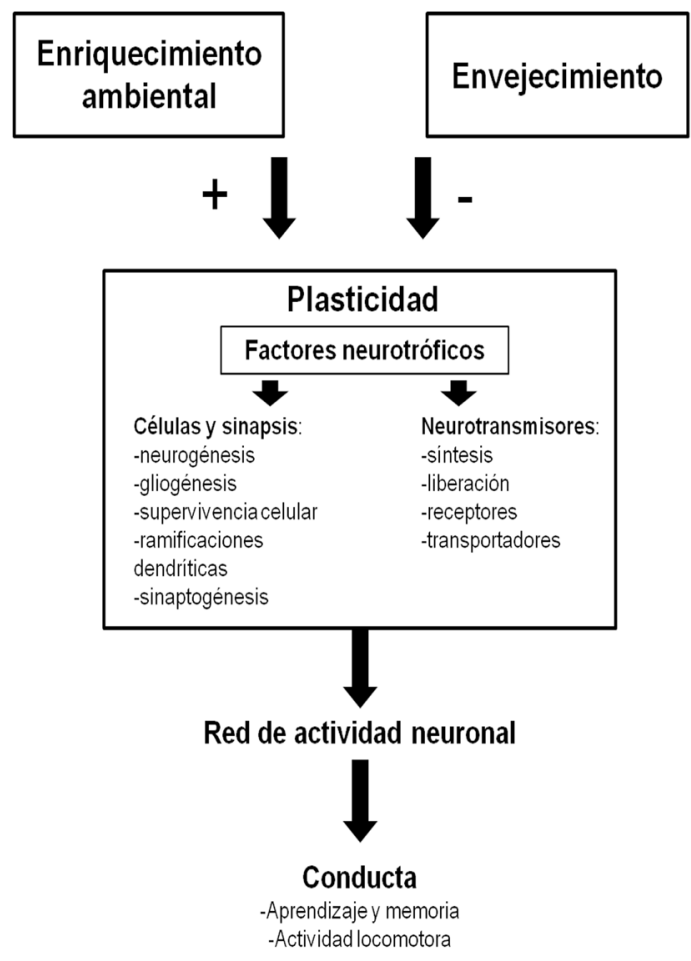

Beneficios del EA en modelos animales de enfermedades neurodegenerativas

La mayoría de los estudios acerca del potencial beneficio del EA sobre modelos transgénicos de enfermedades neurodegenerativas se han realizado en la demencia de Alzheimer, Parkinson y Huntington. Debido a su mayor relación con el proceso de envejecimiento, nos centraremos en las dos primeras.

Alzheimer. La enfermedad de Alzheimer es el tipo de demencia más común. Como se comentó anteriormente, un buen número de estudios epidemiológicos han encontrado una correlación negativa entre la incidencia de esta demencia y el bagaje intelectual, educativo y social del sujeto. Se caracteriza por una importante pérdida neuronal en diferentes regiones cerebrales y un déficit severo en la capacidad de aprendizaje y memoria.

El efecto protector del EA se ha estudiado en modelos animales transgénicos de la enfermedad (TgCRND, APP23, AD11, PDAPP + PS1 ...). Los resultados son generalmente consistentes y encuentran beneficios cognitivos tras la exposición a EA. Por ejemplo, en ratones APP23, el EA mejoró la adquisición de una prueba de memoria espacial (Wolf et al., 2006) y en el modelo AD11, previno los déficits de memoria visual y espacial (Berardi, Braschi, Capsoni, Cattaneo y Maffei, 2007). Del 
mismo modo, en el modelo de ratones PDAPP + PS1, el vivir durante toda su vida en una condición de EA afectó positivamente a su rendimiento en una variedad de pruebas conductuales y redujo el declive cognitivo que ocurre con la edad (Costa et al., 2007). En contraste a la pérdida neuronal que ocurre en esta demencia, 4 meses de EA en ratones TgCRND incrementaron la neurogénesis y sinaptogénesis hippocampal, a pesar de que ya mostraban depósitos de placas amiloides y déficits cognitivos (Herring et al., 2009; Leclerc et al., 1989). Estudios recientes han encontrado también que el EA es capaz de mitigar la patología amiloide (Ambrée et al., 2006; Lazarov et al., 2005), de reducir la hiperfosforilación de la proteína Tau y el estrés oxidativo (Herring et al., 2009; Hu et al., 2010), así como de aliviar la disfunción neurovascular asociada al Alzheimer (Herring et al., 2008). En relación a este último beneficio, el EA estimula la angiogénesis a través de un aumento de la expresión de genes pro-angiogénicos y proteínas (Herring et al., 2008).

Para algunos autores como Kempermann, la neurogénesis estimulada por el EA constituiría el mecanismo que subyace a la relación entre la actividad y la mejora de los síntomas de la demencia Alzheimer (Kempermann, 2008).

No obstante, los resultados acerca del efecto del EA sobre estos modelos no siempre son positivos y dependen de variables como la duración y tipo de EA, así como del modelo transgénico empleado. En el caso de la patología amiloide es donde se observaron los resultados más variables, desde trabajos donde el EA redujo las placas amiloides (Adlar, Perreau, Pop y Cotman, 2005), a otros donde no se observaron cambios (Arendash et al., 2004), o se encontró aumento (Jankowsky et al., 2005).

Parkinson. La demencia de Parkinson es una enfermedad neurodegenerativa que afecta a las neuronas dopaminérgicas de la sustancia negra y causa principalmente alteraciones motoras como temblor. Dos son los principales modelos de ratones transgénicos utilizados para el estudio de esta enfermedad, el modelo 6-OHDA y el MPTP. En estudios recientes, aquellos ratones MPTP que fueron criados en condiciones de EA mostraron ser mucho más resistentes a la neurotoxicidad y su pérdida neuronal fue solo del $40 \%$ en comparación al $75 \%$ de ratones no enriquecidos. Junto a esto, los ratones expuestos a EA mostraron niveles más bajos de transportadores de dopamina en la corteza prefrontal (Zhu, Apparsundaram, Bardo y Dwoskin, 2005) y niveles altos de BDNF en el estriado (Bezard et al., 2003; Faherty, Shepherd, Herasimtschuk y Smeyne, 2005).

\section{Ejercicio aeróbico como uno de los componentes claves del EA}

Una de las principales limitaciones del paradigma de EA es poder aislar el beneficio que aporta cada uno de sus componentes (estimulación sensorial, social, cognitiva y física). La actividad física voluntaria que los animales realizan en las jaulas es el componente que más atención ha recibido ya que por si solo aumenta la expresión de neurogénesis, angiogénesis, de factores neurotróficos, mejora varias funciones cognitivas, sobre todo las dependientes del hipocampo, y reduce los niveles de ansiedad en ratas viejas (Huang et al., 2013; Lista y Sorrentino, 2010; Pietrelli, López-Costa, Goñi, Brusco y Basso, 2012; Sampedro-Piquero, Zancada-Menéndez, Begega, Méndez y Arias, 2013). Evidencia de estudios con humanos y animales sugiere que el ejercicio tiene en general un impacto positivo sobre los aspectos cognitivos y emocionales de la conducta (Cotman y Berchtold, 2002). En modelos genéticos de demencia Alzheimer, la actividad física también ha mostrado un robusto efecto positivo. Por ejemplo, en el modelo THY-Tau22 los ratones que realizaron ejercicio tuvieron un mejor rendimiento en el laberinto en $\mathrm{Y}$, el cual estuvo relacionado con un descenso de los niveles de fosforilación de Tau en el hipocampo (Belarbi et al., 2011). Interesantemente, el ejercicio aumentó los niveles de ARNm de genes que regulan el tráfico del colesterol, lo cual mostraría el potencial beneficioso del ácido graso Omega sobre esta demencia (Elliot, Atlas, Lange y Ginzburg, 2005). En el caso del modelo transgénico de Alzheimer, 3 x Tg-AD, que desarrolla disfunción sináptica y déficits en la potenciación a largo plazo, el ejercicio aeróbico redujo en él marcadores de estrés oxidativo y los déficits en la potenciación a largo plazo (Rodríguez et al., 2011).

En humanos, destaca el trabajo de Erickson y Kramer (2009) en el que ejercicio realizado de forma frecuente y moderada durante 6 meses, mejoró la capacidad atencional, el control ejecutivo, y la actividad cerebral de regiones parietales y frontales en personas mayores. En otro trabajo reciente, estos mismos autores comprobaron que el cerebro de estos sujetos fue más plástico, con un aumento de la conectividad en regiones frontales y temporales, que son de las más sensibles al proceso de envejecimiento. Estos cambios correlacionaron con una mejora de las funciones ejecutivas y posiblemente reflejan una restauración de circuitos neuronales deteriorados con el envejecimiento (McAuley et al., 2011). Curiosamente, la actividad física redujo la probabilidad de declive cognitivo en personas mayores que poseían el alelo E4, pero no en las que no lo poseían (Woodard et al., 2012).

En cuanto a la enfermedad de Parkinson, los estudios sobre el beneficio de la actividad física son más escasos y los resultados contradictorios, a diferencia de lo que ocurre con el EA (Sasco, Paffenbarger, Gendre y Wing, 1992; Thacker et al., 2008). En roedores, el ejercicio realizado antes o después de la degeneración dopaminérgica, mostró beneficios tanto a nivel neuroquímico como conductual. Por ejemplo, actividad física antes, o brevemente después de la administración de una neurotoxina dopaminérgica, puede reducir su efecto, tanto a nivel conductual como en la extensión de la degeneración (Kleim, Jones y Schallert, 2003).

El ejercicio que los animales realizan en las jaulas de enriquecimiento es de tipo voluntario, en cambio otras investigaciones han mostrado que el ejercicio forzado con intensidad 
moderada también tiene beneficios sobre los procesos cognitivos y emocionales de roedores viejos (Sampedro-Piquero et al., 2013). A pesar de las diferencias entre ambos paradigmas de ejercicio, un estudio reciente ha encontrado que el ejercicio forzado y voluntario mejoran por igual la memoria a corto y largo plazo, así como la capacidad de aprendizaje de los animales (Alomari, Khabour, Alzoubi y Alzubi, 2013). Los autores proponen el aumento de los niveles de BDNF como un posible mecanismo que subyace a estos beneficios.

\section{Conclusiones}

El EA es por tanto un paradigma experimental que representa un estilo de vida cognitiva y físicamente activo en humanos. Su aplicación en roedores nos permite ampliar nuestro conocimiento acerca de los mecanismos que subyacen al beneficio físico, social y cognitivo de un estilo de vida activo en personas mayores. Del mismo modo, comprender mejor estos mecanismos en el caso de enfermedades neurodegenerativas, permitirá desarrollar fármacos que mimeticen sus efectos, Environmimetics (McOmish y Hannan, 2007). Debido a la variabilidad de protocolos de EA que existen, muchas cuestiones no han sido todavía resueltas, por ejemplo, ¿cuál es el mejor protocolo y duración para garantizar el máximo beneficio?, ¿cuál es el componente que tiene un mayor impacto en la construcción de la reserva cognitiva? La respuesta a estas cuestiones podría ayudarnos a identificar mejor las poblaciones de sujetos que más se beneficiarían de este tipo de intervenciones.

\section{Referencias}

1. Adlard, P.A., Perreau, V.M., Pop, V. y Cotman, C.W. (2005). Voluntary exercise decreases amyloid load in a transgenic model of Alzheimer's disease. The Journal of Neuroscience, 25, 4217-4221. http://dx.doi.org/10.1523/ JNEUROSCI.0496-05.2005

2. Alomari, M.A., Khabour, O.F., Alzoubi, K.H., Alzubi, M.A. (2013). Forced and voluntary exercises equally improve spatial learning and memory and hippocampal BDNF levels. Behavioral Brain Research, 247, 34-39. http://dx.doi.org/10.1016/j.bbr.2013.03.007

3. Ambrée, O., Leimer, U., Herring, A., Gortz, N., Sachser, N., Heneka, M.T., Paulus, W. y Keyvani, K. (2006). Reduction of amyloid angiopathy and Abeta plaque burden after enriched housing in TgCRND8 mice: involvement of multiple pathways. The American Journal of Pathology 169, 544-552. http://dx.doi.org/10.2353/ajpath.2006.051107

4. Arendash, G.W., García, M.F., Costa, D.A., Cracchiolo, J.R., Wefes, I.M. y Potter, H. (2004). Environmental enrichment improves cognition in aged Alzheimer's transgenic mice despite stable beta-amyloid deposition. Neuroreport, 15, 1751-1754. http://dx.doi.org/10.1097/01. $\underline{\text { wnr.0000137183.68847.4e }}$

5. Belarbi, K., Burnouf, S., Fernández-Gómez, F.J., Laurent, C., Lestavel, S., Figeac, M., Sultan, A., Troquier, L., Leboucher, A., Caillierez, R., Grosjean, M.E., Demeyer,
D., Obriot, H., Brion, I., Barbot, B., Galas, M.C., Staels, B., Humez, S., Sergeant, N., Schraen-Maschke, S., MuhrTailleux, A., Hamdane, M., Buee, L. y Blum, D. (2011). Beneficial effects of exercise in a transgenic mouse model of Alzheimer's disease-like Tau pathology. Neurobiology of Disease, 43, 486-494. http://dx.doi.org/10.1016/j. nbd.2011.04.022

6. Bennett, D.A., Wilson, R.S., Schneider, J.A., Evans, D.A., Mendes de León, C.F., Arnold, S.E., Barnes, L.L. y Bienias, J.L. (2003). Education modifies the relation of AD pathology to level of cognitive function in older persons. Neurology, 60, 1909-1915. http://dx.doi.org/10.1212/01. WNL.0000069923.64550.9F

7. Bennett, J.C., McRae, P.A., Levy, L.J. y Frick, K.M. (2006). Long-term continuous, but not daily, environmental enrichment reduces spatial memory decline in aged male mice. Neurobiology of Learning and Memory, 85, 139-152. http:// dx.doi.org/10.1016/j.nlm.2005.09.003

8. Berardi, N., Braschi, C., Capsoni, S., Cattaneo, A. y Maffei, L. (2007). Environmental enrichment delays the onset of memory deficits and reduces neuropathological hallmarks in a mouse model of Alzheimer-like neurodegeneration. Journal of Alzheimer's Disease, 11, 359-370.

9. Bezard, E., Dovero, S., Belin, D., Duconger, S., JacksonLewis, V., Przedborski, S., Piazza, P.V., Gross, C.E. y Jaber, M. (2003). Enriched environment confers resistance to 1-methyl-4-phenyl-1,2,3,6- tetrahydropyridine and cocaine: involvement of dopamine transporter and trophic factors. The Journal of Neuroscience, 23, 10999-11007.

10. Castilla-Ortega, E., Pedraza, C., Estivill-Torrús, G. y Santín, L.J. (2011). When is adult hippocampal neurogenesis necessary for learning? Evidence from animal research. Reviews in the Neuroscience, 22, 267-283. http://dx.doi. org/10.1515/rns.2011.027

11. Chorpita, B.F. y Barlow, D.H. (1998). The development of anxiety: the role of control in the early environment. Psychological Bulletin, 124, 3-21. http://dx.doi. org/10.1037/0033-2909.124.1.3

12. Costa, D.A., Cracchiolo, J.R., Bachstetter, A.D., Hughes, T.F., Bales, K.R., Paul, S.M., Mervis, R.F., Arendash, G.W. y Potter, H. (2007). Enrichment improves cognition in AD mice by amyloid-related and unrelated mechanisms. Neurobiology of Aging, 28, 831-844. http://dx.doi.org/10.1016/j. neurobiolaging.2006.04.009

13. Cotman, C.W. y Berchtold, N.C. (2002). Exercise: a behavioral intervention to enhance brain health and plasticity. Trends in Neuroscience, 25, 295-301. http://dx.doi. org/10.1016/S0166-2236(02)02143-4

14. Daffner, K.R. (2010) Promoting successful cognitive aging: a comprehensive review. Journal of Alzheimer's Disease, 19, 1101-1122.

15. De Bartolo, P., Leggio, M.G., Mandolesi, L., Foti, F., Gelfo, F., Ferlazzo, F. y Petrosini, L. (2008). Environmental enrichment mitigates the effects of basal forebrain lesions 
on cognitive flexibility. Neuroscience, 154, 444-453. http:// dx.doi.org/10.1016/j.neuroscience.2008.03.069

16. Diniz, D.G., Foro, C.A., Rego, C.M., Gloria, D.A., de Oliveira, F.R., Paes, J.M., de Sousa, A.A., Tokuhashi, T.P., Trindade, L.S., Turiel, M.C., Vasconcelos, E.G., Torres, J.B., Cunnigham, C., Perry, V.H., Vasconcelos, P.F. y Diniz, C.W. (2010). Environmental impoverishment and aging alter object recognition, spatial learning, and dentate gyrus astrocytes. The European Journal of Neuroscience, 32, 509519. http://dx.doi.org/10.1111/j.1460-9568.2010.07296.x

17. Elliott, E., Atlas, R., Lange, A. y Ginzburg, I., 2005. Brain-derived neurotrophic factor induces a rapid dephosphorylation of tau protein through a PI-3 kinase signaling mechanism. The European Journal of Neuroscience, 22, 1081-1089. http://dx.doi.org/10.1111/j.14609568.2005.04290.x

18. Erickson, K.I. y Kramer, A.F. (2009). Aerobic exercise effects on cognitive and neural plasticity in older adults. British Journal of Sports Medicine, 43, 22-24. http://dx.doi. org/10.1136/bjsm.2008.052498

19. Faherty, C.J., Shepherd, K.R., Herasimtschuk, A. y Smeyne, R.J. (2005). Environmental enrichment in adulthood eliminates neuronal death in experimental Parkinsonism, Brain Research, Molecular Brain Research, 134, 170-179. http:// dx.doi.org/10.1016/j.molbrainres.2004.08.008

20. Harati, H., Majchrzak, M., Cosquer, B., Galani, R., Kelche, C., Cassel, J.C. y Barbelivien, A. (2011). Attention and memory in aged rats: impact of lifelong environmental enrichment. Neurobiology of Aging, 32, 718-736. http:// dx.doi.org/10.1016/j.neurobiolaging.2009.03.012

21. Harburger, L.L., Lambert, T.J. y Frick, K.M. (2007). Age-dependent effects of environmental enrichment on spatial reference memory in male mice. Behavioural Brain Research, 185, 43-48. http://dx.doi.org/10.1016/j. bbr.2007.07.009

22. Hebb, D.O. (1947). The effects of early experience on problem solving at maturity. American Psychologist, 2, 306-307.

23. Herring, A., Ambrée, O., Tomm, M., Habermann, H., Sachser, N., Paulus, W. y Keyvani, K. (2009). Environmental enrichment enhances cellular plasticity in transgenic mice with Alzheimer-like pathology. Experimental Neurology, 216,184-192. http://dx.doi.org/10.1016/j.expneurol.2008.11.027

24. Herring, A., Yasin, H., Ambrée, O., Sachser, N., Paulus, W. y Keyvani, K. (2008). Environmental enrichment counteracts Alzheimer's neurovascular dysfunction in TgCRND8 mice. Brain Pathology, 18, 32-39. http://dx.doi. org/10.1111/j.1750-3639.2007.00094.x

25. Hu, Y.S., Xu, P., Pigino, G., Brady, S.T., Larson, J. y Lazarov, O. (2010). Complex environment experience rescues impaired neurogenesis, enhances synaptic plasticity, and attenuates neuropathology in familial Alzheimer's dis- ease-linked APPswe/PS1DeltaE9 mice. FASEB Journal 24, 1667-1681. http://dx.doi.org/10.1096/fj.09-136945

26. Huang, C.X., Qiu, X., Wang, S., Wu, H., Xia, L., Li, C., Gao, Y., Zhang, L., Xiu, Y., Chao, F. y Tang, Y. (2013). Exercise-induced changes of the capillaries in the cortex of middle-aged rats. Neuroscience, 233, 139-145. http:// dx.doi.org/10.1016/j.neuroscience.2012.12.046

27. Jankowsky, J.L., Melnikova, T., Fadale, D.J., Xu, G.M., Slunt, H.H., Gonzáles, V., Youkin, L.H., Youkin, S.G., Borchelt, D.R. y Savonenko, A.V. (2005). Environmental enrichment mitigates cognitive deficits in a mouse model of Alzheimer's disease. The Journal of Neuroscience, 25, 5217-5224. http://dx.doi.org/10.1523/JNEUROSCI.5080-04.2005

28. Katzman, R. (1993). Education and the prevalence of dementia and Alzheimer's disease. Neurology, 43, 13-20. http://dx.doi.org/10.1212/WNL.43.1_Part_1.13

29. Katzman, R., Terry, R., DeTeresa, R., Brown, T., Davies, P., Fuld, P., Renbing, X. y Peck, A. (1988). Clinical, pathological, and neurochemical changes in dementia: a subgroup with preserved mental status and numerous neocortical plaques. Annals of Neurology, 23, 138-144. http://dx.doi. org/10.1002/ana.410230206

30. Kempermann, G. (2008). The neurogenic reserve hypothesis: what is adult hippocampal neurogenesis good for? Trends in Neuroscience, 31,163-169. http://dx.doi. org/10.1016/j.tins.2008.01.002

31. Kempermann, G., Gast, D. y Gage, F.H. (2002). Neuroplasticity in old age: sustained fivefold induction of hippocampal neurogenesis by long-term environmental enrichment. Annals of Neurology, 52, 135-143. http:// dx.doi.org/10.1002/ana.10262

32. Kemppainen, N.M., Aalto, S., Karrasch, M., Någren, K., Savisto, N., Oikonen, V., Viitanen, M., Parkkola, R. y Rinne, J.O. (2008). Cognitive reserve hypothesis: Pittsburgh compound B and fluorodeoxyglucose positron emission tomography in relation to education in mild Alzheimer's disease. Annals of Neurology, 63, 112-118. http://dx.doi. org/10.1002/ana.21212

33. Kleim, J.A., Jones, T.A. y Schallert, T. (2003). Motor enrichment and the induction of plasticity before or after brain injury. Neurochemical Research, 28, 1757-1769. http://dx.doi.org/10.1023/A:1026025408742

34. Kobayashi, S., Ohashi, Y. y Ando, S. (2002). Effects of enriched environments with different durations and starting times on learning capacity during aging in rats assessed by a refined procedure of the Hebb-Williams maze task. The Journal of Neuroscience Research, 70, 340-346. http:// dx.doi.org/10.1002/jnr.10442

35. Lazarov, O., Robinson, J., Tang, Y.P., Hairston, I.S., Korade-Mirnics, Z., Lee, V.M., Hersh, L.B., Sapolsky, R.M., Mirnics, K. y Sisodia, S.S. (2005). Environmental enrichment reduces Abeta levels and amyloid deposi- 
tion in transgenic mice. Cell 120, 701-713. http://dx.doi. org/10.1016/j.cell.2005.01.015

36. Leclerc, N., Beesley, P.W., Brown, I., Colonnier, M., Gurd, J.W., Paladino, T. y Hawkes, R. (1989). Synaptophysin expression during synaptogenesis in the rat cerebellar cortex. The Journal of Comparative Neurology, 280, 197212. http://dx.doi.org/10.1002/cne.902800204

37. Leggio, M.G., Mandolesi, L., Federico, F., Spirito, F., Ricci, B., Gelfo, F. y Petrosini, L. (2005). Environmental enrichment promotes improved spatial abilities and enhanced dendritic growth in the rat. Behavioural Brain Research, 163, 78-90. http://dx.doi.org/10.1016/j.bbr.2005.04.009

38. Li, C., Niu, W., Jiang, CH. y Hu, Y. (2007). Effects of enriched environment on gene expression and signal pathways in cortex of hippocampal CA1 specific NMDAR1 knockout mice. Brain Research Bulletin, 71, 568-577. http://dx.doi.org/10.1016/j.brainresbull.2006.11.011

39. Lista, I. y Sorrentino, G. (2010). Biological mechanisms of physical activity in preventing cognitive decline. Cellular and Molecular Neurobiology, 30, 493e-503. http://dx.doi. org/10.1007/s10571-009-9488-x

40. Mandolesi, L., De Bartolo, P., Foti, F., Gelfo, F., Federico, F., Leggio, M.G. y Petrosini, L. (2008). Environmental enrichment provides a cognitive reserve to be spent in the case of brain lesion. Journal of Alzheimer's Disease, 15, 11-28.

41. McAuley, E., Szabo, A.N., Mailey, E.L., Erickson, K.I., Voss, M., White, S.M., Wójcicki, T.R., Gothe, N., Olson, E.A., Mullen, S.P. y Kramer, A.F. (2011). Non-exercise estimated cardiorespiratory fitness: associations with brain structure, cognition, and memory complaints in older adults. Mental Health and Physical Activity, 4, 5-11. http:// dx.doi.org/10.1016/j.mhpa.2011.01.001

42. McOmish, C.E. y Hannan, A.J. (2007). Enviromimetics: exploring gene environment interactions to identify therapeutic targets for brain disorders. Expert Opinion in Therapeutic Targets, 11, 899-913. http://dx.doi. org/10.1517/14728222.11.7.899

43. Mohammed, A.H., Zhu, S.W., Darmopil, S., Hjerling-Leffler, J., Ernfors, P., Winblad, B., Diamond, M.C., Eriksson, P.S. y Bogdanovic, N. (2002). Environmental enrichment and the brain. Progress in Brain Research, 138, 109-133. http://dx.doi.org/10.1016/S0079-6123(02)38074-9

44. Mora, F., Segovia, G. y del Arco, A. (2007). Aging, plastcity and environmental enrichment: structural changes and neurotransmitter dynamics in several areas of the brain. Brain Research Reviews, 55, 78-88. http://dx.doi.org/10.1016/j. brainresrev.2007.03.011

45. Navarro González, E., Calero García, M.D., Pérez-Díaz, A. y Gómez Ceballos, A.L. (2008). Nivel de independencia en la vida diaria y plasticidad cognitiva en la vejez. Escritos de Psicología, 2, 74-84.

46. Neuropathology Group of the Medical Research Coun- cil Cognitive Function and Ageing Study (MRC CFAS) (2001). Pathological correlates of late-onset dementia in a multicentre community-based population in England and Wales. Lancet, 357, 169-175. http://dx.doi.org/10.1016/ $\underline{\mathrm{S} 0140-6736(00) 03589-3}$

47. Nithianantharajah, J. y Hannan, A.J. (2006). Enriched environments, experience-dependent plasticity and disorders of the nervous system. Nature Reviews. Neuroscience, 7, 697709. http://dx.doi.org/10.1038/nrn1970

48. Petrik, D., Lagace, D.C. y Eisch, A.J. (2012). The neurogenesis hypothesis of affective and anxiety disorders: are we mistaking the scaffolding for the building? Neuropharmacology, 62, 21-34. http://dx.doi.org/10.1016/j.neuropharm.2011.09.003

49. Petrosini, L., De Bartolo, P., Foti, F., Gelfo, F., Cutuli, D., Leggio, MG y Mandolesi, L. (2009). On wheter the environmental enrichment may provide cognitive and brain reserves. Brain Research Reviews, 61, 221-239. http:// dx.doi.org/10.1016/j.brainresrev.2009.07.002

50. Pietrelli, A., López-Costa, R., Goñi, R., Brusco, A. y Basso, A. (2012). Aerobic exercise prevents cognitive decline and reduces anxiety-related behaviours in middle-aged and old rats. Neuroscience, 202, 252-266. http://dx.doi. org/10.1016/j.neuroscience.2011.11.054

51. Richards, M. y Deary, I.J. (2005). A life course approach to cognitive reserve: a model for cognitive aging and development? Annals of Neurology, 58, 617-622. http://dx.doi. org/10.1002/ana.20637

52. Rodríguez, J.J., Noristani, H.N., Olabarria, M., Fletcher, J., Somerville, T.D., Yeh, C.Y. y Verkhratsky, A. (2011). Voluntary running and environmental enrichment restores impaired hippocampal neurogenesis in a triple transgenic mouse model of Alzheimer's disease. Current Alzheimer Research, 8, 707-717. http://dx.doi. org/10.2174/156720511797633214

53. Rosenzweig, M.R., Krech, D., Bennett, E.L. y Zolman, J.F. (1962). Variation in environmental complexity and brain measures. Journal of Comparative \& Physiological Psychology, 55, 1092-1095. http://dx.doi.org/10.1037/ $\underline{\mathrm{h} 0042758}$

54. Sale, A., Berardi, N. y Maffei, L. (2009). Enrich the environment to empower the brain. Trends in Neuroscience, 32, 233-239. http://dx.doi.org/10.1016/j.tins.2008.12.004

55. Sampedro-Piquero, P., Zancada-Menéndez, C., Begega A., Méndez, M. y Arias, J.L. (2013). Effects of forced exercise on spatial memory and cytochrome c oxidase activity in aged rats. Brain Research, 1502, 20-29. http://dx.doi. org/10.1016/j.brainres.2012.12.036

56. Sampedro-Piquero, P., Zancada-Menéndez, C., Begega A., Rubio, S. y Arias, J.L. (2013). Effects of environmental enrichment on anxiety responses, spatial memory and cytochrome c oxidase activity in adult rats. Brain Research Bulletin, http://dx.doi.org/10.1016/j.brainresbull.2013.06.006 
57. Sasco, A.J., Paffenbarger Jr., R.S., Gendre, I. y Wing, A.L., 1992. The role of physical exercise in the occurrence of Parkinson's disease. Archives of Neurology, 49, 360e-365. http://dx.doi.org/10.1001/archneur.1992.00530280040020

58. Scarmeas, N. y Stern, Y. (2003). Cognitive reserve and lifestyle. Journal of Clinical and Experimental Neuropsychology, 25, 625-633. http://dx.doi.org/10.1076/ jcen.25.5.625.14576

59. Schofield, P.W., Logroscino, G., Andrews, H., Albert, S. y Stern, Y. (1997). An association between head circumference and Alzheimer's disease in a population-based study of aging. Neurology, 49, 30-37. http://dx.doi.org/10.1212/ WNL.49.1.30

60. Sisti, H.M., Glass, A.L. y Shors, T.J. (2007). Neurogenesis and the spacing effect, learning over time enhances memory and the survival of new neurons. Learning and memory, 14, 368-375. http://dx.doi.org/10.1101/lm.488707

61. Snowdon, D.A., Kemper, S.J., Mortimer, J.A., Greiner, L.H., Wekstein, D.R. y Markesbery, W.R. (1996). Linguistic ability in early life and cognitive function and Alzheimer's disease in later life. Findings from the Nun Study. JAMA: the journal of the American Medical Association, 275, 528-532. http://dx.doi.org/10.1001/jama.1996.03530310034029

62. Stern, Y. (2002). What is cognitive reserve? Theory and research application of the reserve concept. Journal of the International Neuropsychological Society, 8, 448-460. http://dx.doi.org/10.1017/S1355617702813248

63. Stern, Y. (2009). Cognitive reserve. Neuropsychologia, 47, 2015-2028. http://dx.doi.org/10.1016/j.neuropsychologia.2009.03.004

64. Stern, Y., Alexander, G.E., Prohovnik, I. y Mayeux, R. (1992). Inverse relationship between education and parietotemporal perfusion deficit in Alzheimer's disease. Annals of Neurology, 32, 371-375. http://dx.doi.org/10.1002/ ana.410320311

65. Thacker, E.L., Chen, H., Patel, A.V., McCullough, M.L., Calle, E.E., Thun, M.J., Schwarzschild, M.A. y Ascherio, A. (2008). Recreational physical activity and risk of Parkinson's disease. Movement Disorders, 23, 69-74. http:// dx.doi.org/10.1002/mds. 21772

66. Valenzuela, M.J. y Sachdev, P. (2006). Brain reserve and dementia: A systematic review. Psychological Medicine, 36, 441-454. http://dx.doi.org/10.1017/S0033291705006264
67. Valenzuela, M. y Sachdev, P. (2009). Can cognitive exercise prevent the onset of dementia? Systematic review of randomized clinical trials with longitudinal follow-up. The American Journal of Geriatric Psychiatry, 17, 179-187. http://dx.doi.org/10.1097/JGP.0b013e3181953b57

68. Van Praag, H., Kempermann, G. y Gage, F.H. (2000). Neural consequences of environmental enrichment. Nature Reviews. Neuroscience, 1, 191-198. http://dx.doi. org/10.1038/35044558

69. Williamson, L.L., Chao, A. y Bilbo, S.D. (2012). Environmental enrichment alters glial antigen expression and neuroimmune function in adult rat hippocampus. Brain Behavior and Immunity, 26, 500-510. http://dx.doi. org/10.1016/j.bbi.2012.01.003

70. Wolf, S.A., Kronenberg, G., Lehmann, K., Blankenship, A., Overall, R., Staufenbiel, M. y Kempermann, G. (2006). Cognitive and physical activity differently modulate disease progression in the amyloid precursor protein (APP)-23 model of Alzheimer's disease. Biological Psychiatry, 60, 1314-1323. http://dx.doi.org/10.1016/j. biopsych.2006.04.004

71. Woodard, J.L., Sugarman, M.A., Nielson, K.A., Smith, J.C., Seidenberg, M., Durgerian, S., Butts, A., Hantke, N., Lancaster, M., Matthews, M.A. y Rao, S.M. (2012). Lifestyle and genetic contributions to cognitive decline and hippocampal structure and function in healthy aging. Current Alzheimer Research, 9, 436-446. http://dx.doi. org/10.2174/156720512800492477

72. Zhu, J., Apparsundaram, S., Bardo, M.T. y Dwoskin, L.P. (2005). Environmental enrichment decreases cell surface expression of the dopamine transporter in rat medial prefrontal cortex. Journal of Neurochemistry, 93, 1434-1443. http://dx.doi.org/10.1111/j.1471-4159.2005.03130.x

73. Zimmermann, A., Stauffacher, M., Langhans, W. y Wurbel, H. (2001). Enrichment-dependent differences in novelty exploration in rats can be explained by habituation. Behavioural Brain Research, 121, 11-20. http://dx.doi. org/10.1016/S0166-4328(00)00377-6

Fecha de recepción: 29 de mayo de 2012

Fecha de recepción de la versión modificada: 17 de julio de 2013

Fecha de aceptación: 26 de julio de 2013 